\title{
On Bounding the Union Probability
}

\author{
Jun Yang ${ }^{\star}$, Fady Alajaji ${ }^{\dagger}$ and Glen Takahara ${ }^{\dagger}$ \\ ${ }^{\star}$ Department of Statistical Sciences \\ University of Toronto, Toronto, ON M5S3G3, Canada \\ ${ }^{\dagger}$ Department of Mathematics and Statistics \\ Queen's University, Kingston, ON K7L3N6, Canada \\ E-mails: jun@utstat.toronto.edu; ffady, takahara\}@mast.queensu.ca
}

\begin{abstract}
We present new results on bounding the probability of a finite union of events, $P\left(\bigcup_{i=1}^{N} A_{i}\right)$ for a fixed positive integer $N$, using partial information on the events joint probabilities. We first consider bounds that are established in terms of $\left\{P\left(A_{i}\right)\right\}$ and $\left\{\sum_{j} c_{j} P\left(A_{i} \cap A_{j}\right)\right\}$ where $c_{1}, \ldots, c_{N}$ are given weights. We derive a new class of lower bounds of at most pseudo-polynomial computational complexity. This class of lower bounds generalizes the recent bounds in [1], [2] and can be tighter in some cases than the Gallot-Kounias [3]-[5] and Prékopa-Gao [6] bounds which require more information on the events probabilities. We next consider bounds that fully exploit knowledge of $\left\{P\left(A_{i}\right)\right\}$ and $\left\{P\left(A_{i} \cap A_{j}\right)\right\}$. We establish new numerical lower/upper bounds on the union probability by solving a linear programming problem with $\frac{(N-1)^{3}+N+3}{2}$ variables. These bounds coincide with the optimal lower/upper bounds when $N \leq 7$ and are guaranteed to be sharper than the optimal lower/upper bounds of [1], [2] that use $\left\{P\left(A_{i}\right)\right\}$ and $\left\{\sum_{j} P\left(A_{i} \cap A_{j}\right)\right\}$.
\end{abstract}

Index Terms-Union probability, upper and lower bounds, linear programming, probability of error analysis, communication systems.

\section{INTRODUCTION}

Lower/upper bounds on the union probability $P\left(\bigcup_{i=1}^{N} A_{i}\right)$ in terms of the individual event probabilities $P\left(A_{i}\right)$ 's and the pairwise event probabilities $P\left(A_{i} \cap A_{j}\right)$ 's were actively investigated in the recent past. The optimal bounds can be obtained numerically by solving linear programming (LP) problems with $2^{N}$ variables [6], [7]. Since the number of variables is exponential in the number of events, $N$, some suboptimal but numerically efficient bounds were proposed, such as the bounds in [8] that employ the dual basic feasible solutions to reduce the complexity of the LP problem, and the algorithmic Bonferroni-type lower/upper bounds in [9], [10].

Among the established analytical bounds is the KuaiAlajaji-Takahara lower bound (for convenience, hereafter referred to as the KAT bound) [11] that was shown to be better than the Dawson-Sankoff (DS) [12] and the D. de Caen (DC) [13] bounds. Noting that the KAT bound is expressed in terms of $\left\{P\left(A_{i}\right)\right\}$ and only the sums of the pairwise event probabilities, i.e., $\left\{\sum_{j: j \neq i} P\left(A_{i} \cap A_{j}\right)\right\}$, in order to fully exploit all pairwise event probabilities, it is observed in [14]-[16] that the analytical bounds can be further improved

This work was supported in part by NSERC of Canada. algorithmically by optimizing over subsets. Furthermore, in [6], the KAT bound is extended by using additional partial information such as the sums of joint probabilities of three events, i.e., $\left\{\sum_{j, l} P\left(A_{i} \cap A_{j} \cap A_{l}\right), i=1, \ldots, N\right\}$. Recently, using the same partial information as the KAT bound, i.e., $\left\{P\left(A_{i}\right)\right\}$ and $\left\{\sum_{j: j \neq i} P\left(A_{i} \cap A_{j}\right)\right\}$, the optimal lower/upper bound as well as a new analytical bound which is sharper than the KAT bound were developed in [1], [2].

In this paper, we first establish a new class of lower bounds on $P\left(\bigcup_{i=1}^{N} A_{i}\right)$ using $\left\{P\left(A_{i}\right)\right\}$ and $\left\{\sum_{j} c_{j} P\left(A_{i} \cap A_{j}\right)\right\}$ for a given weight or parameter vector $c=\left(c_{1}, \ldots, c_{N}\right)^{T}$. These lower bounds are shown to have at most pseudopolynomial computational complexity and to be sharper in certain cases than the existing Gallot-Kounias (GK) [3]-[5] and Prékopa-Gao (PG) [6] bounds, although the later bounds employ more information on the events joint probabilities. Furthermore, for bounds on $P\left(\bigcup_{i=1}^{N} A_{i}\right)$ that fully exploit knowledge of $\left\{P\left(A_{i}\right)\right\}$ and $\left\{P\left(A_{i} \cap A_{j}\right)\right\}$, a new numerical lower/upper bound is proposed by solving an LP problem with $\frac{(N-1)^{3}+N+3}{2}$ variables. This numerical lower/upper bound is proven to be an optimal lower/upper bound when $N \leq 7$ and to be always better than the optimal lower/upper bound which uses $\left\{P\left(A_{i}\right)\right\}$ and $\left\{\sum_{j} P\left(A_{i} \cap A_{j}\right)\right\}$. Finally, we should note that these general union probability bounds can be applied to effectively estimate and analyze the error performance of a variety of coded or uncoded communication systems (e.g., see [2], [9], [10], [14], [17]-[22]).

\section{New Bounds using $\left\{\boldsymbol{P}\left(\boldsymbol{A}_{\boldsymbol{i}}\right)\right\}$ AND $\left\{\sum_{j} c_{j} P\left(A_{i} \cap A_{j}\right)\right\}$}

For simplicity, and without loss of generality, we assume the events $\left\{A_{1}, \ldots, A_{N}\right\}$ are in a finite probability space $(\Omega, \mathscr{F}, P)$, where $N$ is a fixed positive integer. Let $\mathscr{B}$ denote the collection of all non-empty subsets of $\{1,2, \ldots, N\}$. Given $B \in \mathscr{B}$, we let $\omega_{B}$ denote the atom in the union $\cup_{i=1}^{N} A_{i}$ such that for all $i=1, \cdots, N, \omega_{B} \in A_{i}$ if $i \in B$ and $\omega_{B} \notin A_{i}$ if $i \notin B$ (note that some of these "atoms" may be the empty set). For ease of notation, for a singleton $\omega \in \Omega$, we denote $P(\{\omega\})$ by $p(\omega)$ and $p\left(\omega_{B}\right)$ by $p_{B}$. Since $\left\{\omega_{B}: i \in B\right\}$ is the collection of all the atoms in $A_{i}$, we have $P\left(A_{i}\right)=\sum_{\omega \in A_{i}} p(\omega)=\sum_{B \in \mathscr{B}: i \in B} p_{B}$, and 


$$
P\left(\bigcup_{i=1}^{N} A_{i}\right)=\sum_{B \in \mathscr{B}} p_{B} .
$$

Suppose there are $N$ functions $f_{i}(B), i=1, \ldots, N$ such that $\sum_{i=1}^{N} f_{i}(B)=1$ for any $B \in \mathscr{B}$ (i.e., for any atom $\left.\omega_{B}\right)$. If we further assume that $f_{i}(B)=0$ if $i \notin B$ (i.e., $\omega_{B} \notin A_{i}$ ), we can write

$P\left(\bigcup_{i=1}^{N} A_{i}\right)=\sum_{B \in \mathscr{B}}\left(\sum_{i=1}^{N} f_{i}(B)\right) p_{B}=\sum_{i=1}^{N} \sum_{B \in \mathscr{B}: i \in B} f_{i}(B) p_{B}$.

Note that if we define

$$
f_{i}(B)= \begin{cases}\frac{1}{|B|}=\frac{1}{\operatorname{deg}\left(\omega_{B}\right)} & \text { if } i \in B \\ 0 & \text { if } i \notin B\end{cases}
$$

where the degree of $\omega, \operatorname{deg}(\omega)$, is the number of $A_{i}$ 's that contain $\omega$, then $\sum_{i=1}^{N} f_{i}(B)=1$ is satisfied and (2) becomes

$$
P\left(\bigcup_{i=1}^{N} A_{i}\right)=\sum_{i=1}^{N} \sum_{\omega \in A_{i}} \frac{p(\omega)}{\operatorname{deg}(\omega)} .
$$

Note that many of the existing bounds, such as the DC bound [13] and KAT bound [11] and the bounds in [1] [2], are based on (4).

In the following lemma, we propose a generalized expression of (4). To the best of our knowledge this lemma is novel.

Lemma 1: Suppose $\left\{\omega_{B}, B \in \mathscr{B}\right\}$ are all the $2^{N}-1$ atoms in $\bigcup_{i} A_{i}$. If $\boldsymbol{c}=\left(c_{1}, \ldots, c_{N}\right)^{T} \in \mathbb{R}^{N}$ satisfies

$$
\sum_{k \in B} c_{k} \neq 0, \quad \text { for all } \quad B \in \mathscr{B}
$$

then we have

$$
\begin{aligned}
P\left(\bigcup_{i=1}^{N} A_{i}\right) & =\sum_{i=1}^{N} \sum_{B \in \mathscr{B}: i \in B} \frac{c_{i} p_{B}}{\sum_{k \in B} c_{k}} \\
& =\sum_{i=1}^{N} \sum_{\omega \in A_{i}} \frac{c_{i} p(\omega)}{\sum_{\left\{k: \omega \in A_{k}\right\}} c_{k}} .
\end{aligned}
$$

Proof: If we define

$$
f_{i}(B)= \begin{cases}\frac{c_{i}}{\sum_{k \in B} c_{k}} & \text { if } i \in B \\ 0 & \text { if } i \notin B\end{cases}
$$

where the parameter vector $\boldsymbol{c}=\left(c_{1}, c_{2}, \ldots, c_{N}\right)^{T}$ satisfies $\sum_{k \in B} c_{k} \neq 0$ for all $B \in \mathscr{B}$ (therefore $c_{i} \neq 0, i=1, \ldots, N$ ), then $\sum_{i} f_{i}(\omega)=1$ holds and we can get (6) from (2).

Note that (6) holds for any $c$ that satisfies (5) and is clearly a generalized expression of (4).

\section{A. Relation to the Cohen-Merhav bound [19]}

Let $m_{i}\left(\omega_{B}\right)$ be non-negative functions. Then by the Cauchy-Schwarz inequality,

$$
\left[\sum_{B: i \in B} f_{i}(B) p_{B}\right]\left[\sum_{B: i \in B} \frac{p_{B}}{f_{i}(B)} m_{i}^{2}\left(\omega_{B}\right)\right] \geq\left[\sum_{B: i \in B} p_{B} m_{i}\left(\omega_{B}\right)\right]^{2} .
$$

Thus, using (2), we have

$$
P\left(\bigcup_{i=1}^{N} A_{i}\right) \geq \sum_{i=1}^{N} \frac{\left[\sum_{B: i \in B} p_{B} m_{i}\left(\omega_{B}\right)\right]^{2}}{\sum_{B: i \in B} \frac{p_{B}}{f_{i}(B)} m_{i}^{2}\left(\omega_{B}\right)} .
$$

If we define $f_{i}(B)$ by (3), then (9) reduces to

$$
P\left(\bigcup_{i=1}^{N} A_{i}\right) \geq \sum_{i} \frac{\left[\sum_{\omega \in A_{i}} p(\omega) m_{i}(\omega)\right]^{2}}{\sum_{j} \sum_{\omega \in A_{i} \cap A_{j}} p(\omega) m_{i}^{2}(\omega)},
$$

which is the Cohen-Merhav lower bound in [19, Theorem 2.1]; note that equality in (10) holds when $m_{i}(\omega)=\frac{1}{\operatorname{deg}(\omega)}$ (i.e., $\left.m_{i}\left(\omega_{B}\right)=\frac{1}{|B|}\right)$.

\section{B. Relation to the GK Bound [3], [4]}

In this subsection, we assume that the elements of $c$ are positive, i.e., $c \in \mathbb{R}_{+}^{N}$, and connect the GK bound [3] [4] with (6). The GK bound was recently revisited in [5] where it is reformulated as

$$
\ell_{\mathrm{GK}}=\max _{c \in \mathbb{R}^{N}} \frac{\left[\sum_{i} c_{i} P\left(A_{i}\right)\right]^{2}}{\sum_{i} \sum_{k} c_{i} c_{k} P\left(A_{i} \cap A_{k}\right)},
$$

and the optimal $\boldsymbol{c}$ for (11), denoted by $\tilde{\boldsymbol{c}}$, can be computed by

$$
\tilde{\boldsymbol{c}}=\boldsymbol{\Sigma}^{-1} \boldsymbol{\alpha},
$$

where $\boldsymbol{\alpha}=\left(P\left(A_{1}\right), \ldots, P\left(A_{N}\right)\right)^{T}$ and $\boldsymbol{\Sigma}$ is the $N \times N$ matrix whose $(i, j)$-th element is $P\left(A_{i} \cap A_{j}\right)$.

First, consider $c \in \mathbb{R}_{+}^{N}$ fixed. Then, by the Cauchy-Schwarz inequality, we have

$$
\left[\sum_{B: i \in B} \frac{c_{i} p_{B}}{\sum_{k \in B} c_{k}}\right]\left[\sum_{B: i \in B}\left(\frac{\sum_{k \in B} c_{k}}{c_{i}}\right) p_{B}\right] \geq P\left(A_{i}\right)^{2} .
$$

Note that

$$
\begin{aligned}
\sum_{B: i \in B}\left(\frac{\sum_{k \in B} c_{k}}{c_{i}}\right) p_{B} & =\frac{1}{c_{i}} \sum_{k=1}^{N} \sum_{B: i \in B, k \in B} c_{k} p_{B} \\
& =\frac{\sum_{k} c_{k} P\left(A_{i} \cap A_{k}\right)}{c_{i}} .
\end{aligned}
$$

Then for all $i$,

$$
\sum_{B: i \in B} \frac{c_{i} p_{B}}{\sum_{k \in B} c_{k}} \geq \frac{c_{i}^{2} P\left(A_{i}\right)^{2}}{c_{i} \sum_{k} c_{k} P\left(A_{i} \cap A_{k}\right)}
$$

By summing (15) over $i$, we get another new lower bound:

$$
P\left(\bigcup_{i} A_{i}\right) \geq \sum_{i=1}^{N} \frac{c_{i}^{2} P\left(A_{i}\right)^{2}}{c_{i} \sum_{k} c_{k} P\left(A_{i} \cap A_{k}\right)} .
$$

Note that we can use Cauchy-Schwarz Inequality again:

$$
\begin{aligned}
& {\left[\sum_{i=1}^{N} \frac{c_{i}^{2} P\left(A_{i}\right)^{2}}{c_{i} \sum_{k} c_{k} P\left(A_{i} \cap A_{k}\right)}\right]\left[\sum_{i} c_{i} \sum_{k} c_{k} P\left(A_{i} \cap A_{k}\right)\right]} \\
& \geq\left[\sum_{i} c_{i} P\left(A_{i}\right)\right]^{2} .
\end{aligned}
$$


Since the above inequality holds for any positive $c$, we have

$$
\begin{aligned}
P\left(\bigcup_{i} A_{i}\right) & \geq \max _{c \in \mathbb{R}_{+}^{N}} \sum_{i=1}^{N} \frac{c_{i}^{2} P\left(A_{i}\right)^{2}}{c_{i} \sum_{k} c_{k} P\left(A_{i} \cap A_{k}\right)} \\
& \geq \max _{c \in \mathbb{R}_{+}^{N}} \frac{\left[\sum_{i} c_{i} P\left(A_{i}\right)\right]^{2}}{\sum_{i} \sum_{k} c_{i} c_{k} P\left(A_{i} \cap A_{k}\right)} .
\end{aligned}
$$

Note that the lower bounds in (18) are weaker than the GK bound (11), however, if the optimal $c$ of (11), $\tilde{\boldsymbol{c}}$, happen to satisfy $\tilde{\boldsymbol{c}} \in \mathbb{R}_{+}^{N}$, then the bounds in (18) coincide with the GK bound (11).

\section{New Class of Lower Bounds}

We only consider $c \in \mathbb{R}_{+}^{N}$ in this subsection. A new class of lower bounds is given in the following theorem.

Theorem 1: Defining $\mathscr{B}^{-}=\mathscr{B} \backslash\{1, \ldots, N\}, \tilde{\gamma}_{i}:=$ $\sum_{k} c_{k} P\left(A_{i} \cap A_{k}\right), \tilde{\alpha}_{i}:=P\left(A_{i}\right)$ and

$$
\tilde{\delta}:=\max _{i}\left[\frac{\tilde{\gamma}_{i}-\left(\sum_{k} c_{k}-\min _{k} c_{k}\right) \tilde{\alpha}_{i}}{\min _{k} c_{k}}\right]^{+},
$$

where $c \in \mathbb{R}_{+}^{N}$, a class of lower bounds is given by

$$
P\left(\bigcup_{i=1}^{N} A_{i}\right) \geq \tilde{\delta}+\sum_{i=1}^{N} \ell_{i}^{\prime}(\boldsymbol{c}, \tilde{\delta}),
$$

where

$$
\begin{aligned}
\ell_{i}^{\prime}(\boldsymbol{c}, x)= & {\left[P\left(A_{i}\right)-x\right]\left(\frac{c_{i}}{\sum_{k \in B_{1}^{(i)}} c_{k}}+\frac{c_{i}}{\sum_{k \in B_{2}^{(i)}} c_{k}}\right.} \\
& \left.-\frac{c_{i} \sum_{k} c_{k}\left[P\left(A_{i} \cap A_{k}\right)-x\right]}{\left[P\left(A_{i}\right)-x\right]\left(\sum_{k \in B_{1}^{(i)}} c_{k}\right)\left(\sum_{k \in B_{2}^{(i)}} c_{k}\right)}\right),
\end{aligned}
$$

and

$$
\begin{aligned}
& B_{1}^{(i)}=\arg \max _{\{B \in \mathscr{B}:: i \in B\}} \frac{\sum_{k \in B} c_{k}}{c_{i}} \\
& \text { s.t. } \quad \frac{\sum_{k \in B} c_{k}}{c_{i}} \leq \frac{\sum_{k} c_{k}\left[P\left(A_{i} \cap A_{k}\right)-x\right]}{c_{i}\left[P\left(A_{i}\right)-x\right]}, \\
& B_{2}^{(i)}=\arg \min _{\{B \in \mathscr{B}:: i \in B\}} \frac{\sum_{k \in B} c_{k}}{c_{i}} \\
& \text { s.t. } \quad \frac{\sum_{k \in B} c_{k}}{c_{i}} \geq \frac{\sum_{k} c_{k}\left[P\left(A_{i} \cap A_{k}\right)-x\right]}{c_{i}\left[P\left(A_{i}\right)-x\right]} .
\end{aligned}
$$

Proof: Let $x=p_{\{1,2, \ldots, N\}}$ and consider $\sum_{i} \ell_{i}^{\prime}(\boldsymbol{c}, x)+$ $x$ as a new lower bound where where $\ell_{i}^{\prime}(\boldsymbol{c}, x)$ equals to the objective value of the problem

$$
\begin{aligned}
& \min _{\left\{p_{B}: i \in B, B \in \mathscr{B}^{-}\right\}} \sum_{B: i \in B, B \in \mathscr{B}^{-}} \frac{c_{i} p_{B}}{\sum_{k \in B} c_{k}} \\
& \text { s.t. } \sum_{B: i \in B, B \in \mathscr{B}^{-}} p_{B}=P\left(A_{i}\right)-x, \\
& \quad \sum_{B: i \in B, B \in \mathscr{B}^{-}}\left(\frac{\sum_{k \in B} c_{k}}{c_{i}}\right) p_{B}=\frac{1}{c_{i}} \sum_{k} c_{k}\left[P\left(A_{i} \cap A_{k}\right)-x\right], \\
& p_{B} \geq 0, \text { for all } B \in \mathscr{B}^{-} \text {such that } i \in B .
\end{aligned}
$$

The solution of (23) exists if and only if

$$
\min _{k} c_{k} \leq \frac{\tilde{\gamma}_{i}-\left(\sum_{k} c_{k}\right) x}{\tilde{\alpha}_{i}-x} \leq \sum_{k} c_{k}-\min _{k} c_{k}
$$

Therefore, the new lower bound can be written as

$$
\begin{aligned}
& \min _{x}\left[x+\sum_{i=1}^{N} \ell_{i}^{\prime}(\boldsymbol{c}, x)\right] \quad \text { s.t. } \\
& {\left[\frac{\tilde{\gamma}_{i}-\left(\sum_{k} c_{k}-\min _{k} c_{k}\right) \tilde{\alpha}_{i}}{\min _{k} c_{k}}\right]^{+} \leq x \leq \frac{\tilde{\gamma}_{i}-\left(\min _{k} c_{k}\right) \tilde{\alpha}_{i}}{\sum_{k} c_{k}-\min _{k} c_{k}}, \forall i .}
\end{aligned}
$$

We can prove that the objective function of (25) is nondecreasing with $x$. Therefore, defining $\tilde{\delta}$ as in (19), the new lower bound can be written as (20) where $\ell_{i}^{\prime}(\boldsymbol{c}, \tilde{\delta})$ can be obtained by solving (23), which is given in (21).

Remark 1: Note that the problems in (22) are exactly the 0/1 knapsack problem with mass equals to value [23], which can be computed in pseudo-polynomial time, and can be arbitrarily closely approximated by an algorithm running in polynomial time [23].

Remark 2: It can readily be shown that if $c=\kappa \mathbf{1}$ for any non-zero constant $\kappa$ with 1 being the all-one vector of length $N$, the new lower bound reduces to the analytical lower bound in [1], [2], which is sharper than the KAT bound. It can also be shown that if the optimal $\tilde{\boldsymbol{c}}$ of the GK bound satisfies $\tilde{\boldsymbol{c}} \in \mathbb{R}_{+}^{N}$, then the new lower bound is sharper than the GK bound.

\section{New Bounds using $\left\{\boldsymbol{P}\left(\boldsymbol{A}_{\boldsymbol{i}}\right)\right\}$ and $\left\{\boldsymbol{P}\left(\boldsymbol{A}_{\boldsymbol{i}} \cap \boldsymbol{A}_{\boldsymbol{j}}\right)\right\}$}

In this section, we derive new numerical lower/upper bounds for $P\left(\bigcup_{i=1}^{N} A_{i}\right)$ using $\left\{P\left(A_{i}\right)\right\}$ and $\left\{P\left(A_{i} \cap A_{j}\right)\right\}$. First, consider the $p_{B}$ 's in (1) as variables. Then the following (exhaustive) LP problem with $2^{N}$ variables gives the optimal lower/upper bound established using $\left\{P\left(A_{i}\right)\right\}$ and $\left\{P\left(A_{i} \cap\right.\right.$ $\left.\left.A_{j}\right)\right\}$ :

$$
\begin{array}{ll}
\min _{\left\{p_{B}, B \in \mathscr{B}\right\}} / \max _{\left\{p_{B}, B \in \mathscr{B}\right\}} \sum_{B \in \mathscr{B}} p_{B} \\
\text { s.t. } & \sum p_{B, j \in B, B \in \mathscr{B}} p_{B}=P\left(A_{i} \cap A_{j}\right), \quad i, j \in\{1, \ldots, N\}, \\
& p_{B} \geq 0, B \in \mathscr{B} .
\end{array}
$$

, The optimality of (26) can be easily proved by showing its achievability: for each $p_{B}$, construct an atom $\omega_{B}$ such that $p\left(\omega_{B}\right)=p_{B}$ and let $\omega_{B} \in A_{i}, \forall i \in B$. However, the computational complexity of the optimal lower/upper bound 
in (26) is exponential. Next, we consider a relaxed problem of (26), which is given in the following:

$$
\begin{aligned}
& \min _{\left\{p_{B}, B \in \mathscr{B}\right\}} / \max _{\left\{p_{B}, B \in \mathscr{B}\right\}} \sum_{B \in \mathscr{B}} p_{B}, \\
& \text { s.t. } \quad \sum_{i, j \in B, B \in \mathscr{B}} p_{B}=P\left(A_{i} \cap A_{j}\right), \quad i, j \in\{1, \ldots, N\}, \\
& \sum_{B: i, j, l \in B,|B|=k} p_{B} \geq 0, \quad \sum_{B: i, j \in B, l \notin B,|B|=k} p_{B} \geq 0, \\
& \sum_{B: i \in B, j, l \notin B,|B|=k} p_{B} \geq 0, \quad \sum_{B: i, j, l \notin B,|B|=k} \geq 0, \\
& \forall i, j, l, k \in\{1, \ldots, N\} .
\end{aligned}
$$

Since the solution of (27) is a lower/upper bound for the union probability $P\left(\bigcup_{i=1}^{N} A_{i}\right)$, we next show that the solution of (27) can be obtained by solving an LP problem with $\frac{(N-1)^{3}+N+3}{2}$ variables, which coincides with the optimal lower/upper bounds when $N \leq 7$. The main results are in the following.

Lemma 2: The solution of problem (27) coincides with the optimal lower/upper bound in (26) when $N \leq 7$.

Lemma 3: The problem (27) shares the same solution with the following LP:

$$
\begin{aligned}
& \min _{\left\{p_{B}, B \in \mathscr{B}\right\}} / \max _{\left\{p_{B}, B \in \mathscr{B}\right\}} \sum_{B \in \mathscr{B}} p_{B}, \\
& \sum_{i, j \in B, B \in \mathscr{B}} p_{B}=P\left(A_{i} \cap A_{j}\right), \quad i, j \in\{1, \ldots, N\}, \\
& \sum_{B: i, j, l \in B,|B|=k} p_{B}+\sum_{B: i, j \in B, l \notin B,|B|=k} p_{B} \geq 0, \\
& \sum_{B: l \in B, i, j \notin B,|B|=k} p_{B}+\sum_{B: i, j, l \notin B,|B|=k} p_{B} \geq 0, \\
& \sum_{B: i, j, l \in B,|B|=k} p_{B}+\sum_{B: i, j, l \notin B,|B|=k} \geq 0, \\
& \sum_{B: i, j \in B,|B|=k} p_{B}+\sum_{B: l \in B, i, j \notin B,|B|=k} p_{B} \geq 0, \\
& \forall i, j, l, k \in\{1, \ldots, N\} . \quad \sum_{B: i \in B, j, l \notin B,|B|=k} p_{B} \geq 0,
\end{aligned}
$$

Theorem 2: Defining $a_{i j}(k)=\sum_{i, j \in B,|B|=k} p_{B}$, the LP problem (28) can be reformulated as an LP of $\left\{a_{i j}(k)\right\}$ (i.e., $N^{3}$ variables). The number of variables can hence be reduced from $N^{3}$ to $\frac{(N-1)^{3}+N+3}{2}$.

Proof: Define $a(k)=\sum_{|B|=k} p_{B}$ and $a_{i}(k)=$ $\sum_{i \in B,|B|=k} p_{B}$, then it can be readily shown that $a(k)=$ $\sum_{i=1}^{N} \frac{a_{i}(k)}{k}$ and $a_{i}(k)=\sum_{j=1}^{N} \frac{a_{i j}(k)}{k}$. Therefore, both $a(k)$ and $a_{i}(k)$ are linear functions of $\left\{a_{i j}(k)\right\}$.

We next demonstrate that the number of variables can be reduced from $N^{3}$ to $\frac{(N-1)^{3}+N+3}{2}$. Note that according to the definition of $a_{i j}(k)$, we have: i) $a_{i j}(1)=$
$P\left(\left\{x \in A_{i} \cap A_{j}, \operatorname{deg}(x)=1\right\}\right)=0, \forall i \neq j$; ii) $a_{i j}(k)=$ $a_{j i}(k)$; iii) $a_{i j}(N)=P\left(\bigcap_{i=1}^{N} A_{i}\right)$ for any $i$ and $j$. Therefore, the number of variables for different values of $k$ can be reduced to

$$
\begin{cases}N & \text { if } k=1 \\ \frac{N(N-1)}{2} & \text { if } k=2, \ldots, N-1 \\ 1 & \text { if } k=N\end{cases}
$$

Thus, the total number of variables is $N+\frac{N(N-1)(N-2)}{2}+1$.

Now it is suffices to show that the objective function and all the constraints in (28) can be written as functions of $a_{i j}(k)$ so that all $\left\{p_{B}\right\}$ can be replaced using $a_{i j}(k)$. In the following, we directly give the results, which one can easily verify.

The objective function and the first constraint of (28) can be written as

$$
\begin{aligned}
& \sum_{k} \sum_{i} \sum_{j} \frac{a_{i j}(k)}{k^{2}}=\sum_{B \in \mathscr{B}} p_{B}, \\
& \sum_{k} a_{i j}(k)=\sum_{i, j \in B, B \in \mathscr{B}} p_{B}=P\left(A_{i} \cap A_{j}\right), \quad \forall i, j .
\end{aligned}
$$

Finally, for all $i, j, l, k \in\{1, \ldots, N\}$, the other constraints of (28) as functions of $\left\{p_{B}\right\}$ can be written as functions of $\left\{a_{i j}(k)\right\}$ as follows:

$$
\begin{aligned}
a_{i j}(k) & =\sum_{B: i, j, l \in B,|B|=k} p_{B}+\sum_{B: i, j \in B, l \notin B,|B|=k} p_{B}, \\
a(k)- & a_{i}(k)-a_{j}(k)+a_{i j}(k) \\
= & \sum_{B: l \in B, i, j \notin B,|B|=k} p_{B}+\sum_{B: i, j, l \notin B,|B|=k} p_{B}, \\
a(k)- & a_{l}(k)-a_{i}(k)-a_{j}(k)+a_{i j}(k)+a_{i l}(k)+a_{j l}(k) \\
= & \sum_{B: i, j, l \in B,|B|=k} p_{B}+\sum_{B: i, j, l \notin B,|B|=k} p_{B}, \\
a_{l}(k)+ & a_{i j}(k)-a_{i l}(k)-a_{j l}(k) p_{B}+\sum_{B: l \in B, i, j \notin B,|B|=k} p_{B}, \\
& \sum_{B: i, j \in B, l \notin B,|B|=k} \sum_{B: i, l \in B, j \notin B,|B|=k} p_{B: i \in B, j, l \notin B,|B|=k} .
\end{aligned}
$$

Therefore, the lower/upper bounds of (27) can be solved by an LP with $\frac{(N-1)^{3}+N+3}{2}$ variables.

Remark 3: According to Lemma 2, the new numerical lower/upper bound coincides with the optimal lower/upper bounds in (26) when $N \leq 7$. Furthermore, we can show that the new numerical lower/upper bounds are sharper than the numerical bounds in [1], [2], which have been proved to be the optimal lower/upper bounds in terms of $\left\{P\left(A_{i}\right)\right\}$ and $\left\{\sum_{j} P\left(A_{i} \cap A_{j}\right)\right\}$.

\section{NUMERICAL EXAMPLES}

Due to the space limitation, we only present lower bounds in this section. The same eight systems as in [1] are used and the corresponding results are shown in Table I. For comparison, we include bounds that utilize $\left\{P\left(A_{i}\right)\right\}$ and 
TABLE I

COMPARISON OF LOWER BOUNDS (* INDICATES $\tilde{\boldsymbol{c}} \in \mathbb{R}_{+}^{N}$ AND A BOLD NUMBER INDICATES COINCIDENCE WITH THE OPTIMAL BOUND (26)).

\begin{tabular}{|c||c|c|c|c|c|c|c|c|}
\hline System & I & II* $^{*}$ & III* $^{*}$ & IV & V & VI & VII & VIII* \\
\hline \hline$N$ & 6 & 6 & 6 & 7 & 3 & 4 & 4 & 4 \\
\hline$P\left(\bigcup_{i=1}^{N} A_{i}\right)$ & 0.7890 & 0.6740 & 0.7890 & 0.9687 & 0.3900 & 0.3252 & 0.5346 & 0.5854 \\
\hline KAT Bound [11] & 0.7247 & 0.6227 & 0.7222 & 0.8909 & 0.3833 & 0.2769 & 0.4434 & 0.5412 \\
\hline GK Bound [3], [4] & 0.7601 & 0.6510 & 0.7508 & 0.9231 & 0.3813 & 0.2972 & 0.4750 & 0.5390 \\
\hline PG Bound [6] & 0.7443 & 0.6434 & 0.7556 & 0.9148 & $\mathbf{0 . 3 9 0 0}$ & 0.3240 & 0.5281 & 0.5726 \\
\hline Analytical Bound [2, Eq. (7)] & 0.7247 & 0.6227 & 0.7222 & 0.8909 & $\mathbf{0 . 3 9 0 0}$ & 0.3205 & 0.4562 & 0.5464 \\
\hline Numerical Bound [2, Eq. (5)] & 0.7487 & 0.6398 & 0.7427 & 0.9044 & $\mathbf{0 . 3 9 0 0}$ & $\mathbf{0 . 3 2 5 2}$ & $\mathbf{0 . 5 0 9 0}$ & 0.5531 \\
\hline New Bound (20) with $\boldsymbol{c}=\tilde{c}^{+}$ & 0.7638 & 0.6517 & 0.7512 & 0.9231 & $\mathbf{0 . 3 9 0 0}$ & 0.2951 & 0.4905 & 0.5412 \\
\hline New Bound (20) with random $\boldsymbol{c}$ & 0.7783 & 0.6633 & 0.7810 & 0.9501 & $\mathbf{0 . 3 9 0 0}$ & 0.3203 & 0.4992 & 0.5666 \\
\hline Stepwise Bound [9] & $\mathbf{0 . 7 8 9 0}$ & $\mathbf{0 . 6 7 4 0}$ & $\mathbf{0 . 7 8 9 0}$ & $\mathbf{0 . 9 6 8 7}$ & $\mathbf{0 . 3 9 0 0}$ & 0.3027 & 0.5009 & $\mathbf{0 . 5 6 7 3}$ \\
\hline New Numerical Bound (27) & $\mathbf{0 . 7 8 9 0}$ & $\mathbf{0 . 6 7 4 0}$ & $\mathbf{0 . 7 8 9 0}$ & $\mathbf{0 . 9 6 8 7}$ & $\mathbf{0 . 3 9 0 0}$ & $\mathbf{0 . 3 2 5 2}$ & $\mathbf{0 . 5 0 9 0}$ & $\mathbf{0 . 5 6 7 3}$ \\
\hline
\end{tabular}

$\left\{\sum_{j} P\left(A_{i} \cap A_{j}\right), i=1, \ldots, N\right\}$, such as the KAT bound [11], the analytical bound in [1], [2], and the numerical optimal bound in this class [1], [2]. We also include the GK bound [3], [4] and the stepwise bound [9], which fully exploit $\left\{P\left(A_{i}\right)\right\}$ and $\left\{P\left(A_{i} \cap A_{j}\right)\right\}$. The PG lower bound [6], which extends the KAT bound by using $\left\{P\left(A_{i}\right)\right\},\left\{\sum_{j} P\left(A_{i} \cap A_{j}\right)\right\}$ and $\left\{\sum_{j, l} P\left(A_{i} \cap A_{j} \cap A_{l}\right)\right\}$, is also investigated in the examples. The Cohen-Merhav bound (10) [19] is not included since it is not clear how to choose the function $m_{i}(\omega)$ in our examples.

For the proposed bound (20) we consider two cases for choosing $\boldsymbol{c}$. The first choice for $\boldsymbol{c}$, denoted by $\tilde{\boldsymbol{c}}^{+}$, has components $\tilde{c}_{i}^{+}=\max \left(\tilde{c}_{i}, \epsilon\right)$ with $\tilde{\boldsymbol{c}}$ given in (12) and $\epsilon>0$ close to zero. Therefore, if $\tilde{\boldsymbol{c}} \in \mathbb{R}_{+}^{N}$ then $\tilde{\boldsymbol{c}}^{+}=\tilde{\boldsymbol{c}}$, so that in this case the new bound (20) is guaranteed to be sharper than the GK bound. If $\tilde{\boldsymbol{c}} \notin \mathbb{R}_{+}^{N}$, on the other hand, we still have $\tilde{\boldsymbol{c}}^{+} \in \mathbb{R}_{+}^{N}$. The second choice of $\boldsymbol{c}$ is to randomly generate $c \in \mathbb{R}_{+}^{N}$ and compute (20). In the examples, we generate 1000 values for $c$ and show the largest obtained value for (20).

From Table I, one remarks that for Systems II, III and VIII we have $\tilde{\boldsymbol{c}} \in \mathbb{R}_{+}^{N}$, so that the new bound (20) with $\boldsymbol{c}=\tilde{\boldsymbol{c}}$ is sharper than the GK bound, as expected. Also, the new bound (20) can be further improved by randomly generating additional $c$ values as shown in the table. Furthermore, the PG bound which uses sums of joint probabilities of three events, may be even poorer (e.g., see Systems I and VI) than the numerical bound in [1], [2] which utilizes less information but is optimal in the class of lower bounds using $\left\{P\left(A_{i}\right)\right\}$ and $\left\{\sum_{j} P\left(A_{i} \cap A_{j}\right)\right\}$. It is also weaker than (20) in several cases (see Systems I-IV). Finally, our numerical bound (27) is always sharper than the other tested bounds, and coincides with the optimal bound (26) with exponential complexity in $N$ since $N<7$ holds for these examples.

\section{REFERENCES}

[1] J. Yang, F. Alajaji, and G. Takahara, "Lower bounds on the probability of a finite union of events," submitted, 2014. [Online]. Available: http://arxiv.org/abs/1401.5543

[2] - "New bounds on the probability of a finite union of events," in 2014 IEEE International Symposium on Information Theory (ISIT), June 2014, pp. 1271-1275.

[3] S. Gallot, "A bound for the maximum of a number of random variables," Journal of Applied Probability, vol. 3, no. 2, pp. 556-558, 1966.

[4] E. G. Kounias, "Bounds for the probability of a union, with applications," The Annals of Mathematical Statistics, vol. 39, no. 6, pp. 21542158, 1968.
[5] C. Feng, L. Li, and J. Shen, "Some inequalities in functional analysis, combinatorics, and probability theory," The Electronic Journal of Combinatorics, vol. 17, no. R58, p. 1, 2010.

[6] A. Prékopa and L. Gao, "Bounding the probability of the union of events by aggregation and disaggregation in linear programs," Discrete Applied Mathematics, vol. 145, no. 3, pp. 444-454, 2005.

[7] P. Veneziani, "Lower bounds of degree 2 for the probability of the union of $\mathrm{N}$ events via linear programming," June 2007, unpublished.

[8] B. Vizvári, "New upper bounds on the probability of events based on graph structures," RUTCOR Research Report (Sept. 2004).

[9] H. Kuai, F. Alajaji, and G. Takahara, "Tight error bounds for nonuniform signaling over AWGN channels," IEEE Transactions on Information Theory, vol. 46, no. 7, pp. 2712-2718, 2000.

[10] F. Behnamfar, F. Alajaji, and T. Linder, "Tight error bounds for spacetime orthogonal block codes under slow Rayleigh flat fading," IEEE Transactions on Communications, vol. 53, no. 6, pp. 952-956, 2005.

[11] H. Kuai, F. Alajaji, and G. Takahara, "A lower bound on the probability of a finite union of events," Discrete Mathematics, vol. 215, no. 1-3, pp. $147-158,2000$.

[12] D. Dawson and D. Sankoff, "An inequality for probabilities," Proceedings of the American Mathematical Society, vol. 18, no. 3, pp. 504-507, 1967.

[13] D. De Caen, "A lower bound on the probability of a union," Discrete Mathematics, vol. 169, no. 1, pp. 217-220, 1997.

[14] F. Behnamfar, F. Alajaji, and T. Linder, "An efficient algorithmic lower bound for the error rate of linear block codes," IEEE Transactions on Communications, vol. 55, no. 6, pp. 1093-1098, 2007.

[15] F. M. Hoppe, "Improving probability bounds by optimization over subsets," Discrete Mathematics, vol. 306, no. 5, pp. 526-530, 2006.

[16] — , "The effect of redundancy on probability bounds," Discrete Mathematics, vol. 309, no. 1, pp. 123-127, 2009.

[17] G. Seguin, "A lower bound on the error probability for signals in white Gaussian noise," IEEE Transactions on Information Theory, vol. 44, no. 7, pp. 3168-3175, Nov. 1998.

[18] S. Yousefi and A. K. Khandani, "A new upper bound on the ML decoding error probability of linear binary block codes in AWGN interference," IEEE Trans. Inf. Theory, vol. 50, no. 12, pp. 3026-3036, Dec. 2004.

[19] A. Cohen and N. Merhav, "Lower bounds on the error probability of block codes based on improvements on de caen's inequality," IEEE Transactions on Information Theory, vol. 50, no. 2, pp. 290-310, 2004.

[20] H. Nguyen and N. Tran, "Bonferroni-type bounds for CDMA systems with nonuniform signalling," IEEE Commun. Lett., vol. 9, no. 7, pp. 583-585, July 2005.

[21] R. Bettancourt, L. Szczecinski, and R. Feick, "BER evaluation of BICMID via Bonferroni-type bounds," IEEE Trans. Veh. Technol., vol. 57, no. 5, pp. 2815-2821, Sep. 2008

[22] Z. Mao, J. Cheng, and J. Shen, "A new lower bound on error probability for nonuniform signals over AWGN channels," in Wireless Communications and Networking Conference (WCNC). IEEE, 2013, pp. 30053009.

[23] V. V. Vazirani, Approximation Algorithms. New York, NY, USA: Springer-Verlag New York, Inc., 2001. 